\title{
The multiperiodic $\delta$ Scuti star 4 Canum Venaticorum: 1997 APT photometry
}

\author{
M. Breger and F. Hiesberger \\ Astronomisches Institut der Universität Wien, Türkenschanzstr. 17, A-1180 Wien, Austria \\ e-mail: breger@astro.univie.ac.at
}

Received November 2; accepted November 30, 1998

\begin{abstract}
. 204 hours of high-quality measurements of 4 CVn during 32 nights were obtained with the $0.75 \mathrm{~m}$ Automatic Photolectric Telescope (APT). The two comparison stars measured agreed to \pm 2.7 mmag per single measurement in Stromgren $y$ and $\pm 3.0 \mathrm{mmag}$ in $v$. It is shown that APT's can be used successfully for the study of stellar nonradial pulsation at the millimag level.

An analysis of the multiperiodicity of $4 \mathrm{CVn}$ revealed the presence of 19 simultaneously excited pulsation frequencies. This presents a considerable increase from the 7 frequencies found during a previous campaign of the Delta Scuti Network. The previously known frequencies were confirmed with amplitude variability on a time scale of years.
\end{abstract}

Key words: stars: $\delta$ Sct - stars: oscillations - stars: individual: $4 \mathrm{CVn}$ - techniques: photometric

\section{Introduction}

Asteroseismology of $\delta$ Scuti stars has reached a stage where the choice between different models of stellar structure and evolution require a large number of known pulsation frequencies. Recent examples of comparisons between observed and modelled nonradial multiple frequencies can be found in Pamyatnykh et al. (1998), Breger et al. (1998), Viskum et al. (1998) and Guzik, Templeton \& Bradley (1998). The detection of the frequencies presents a severe challenge to the observers: the most commonly applied method utilizes extensive multisite campaigns, where millimag photometric accuracy is obtained. To obtain the required instrumental stability, the Delta Scuti Network adopts the three-star technique (Breger 1993), where measurements of the variable star are alternated with those of two comparison stars. The stars are usually changed manually at the telescope approximately every $90 \mathrm{~s}$.

Send offprint requests to: M. Breger

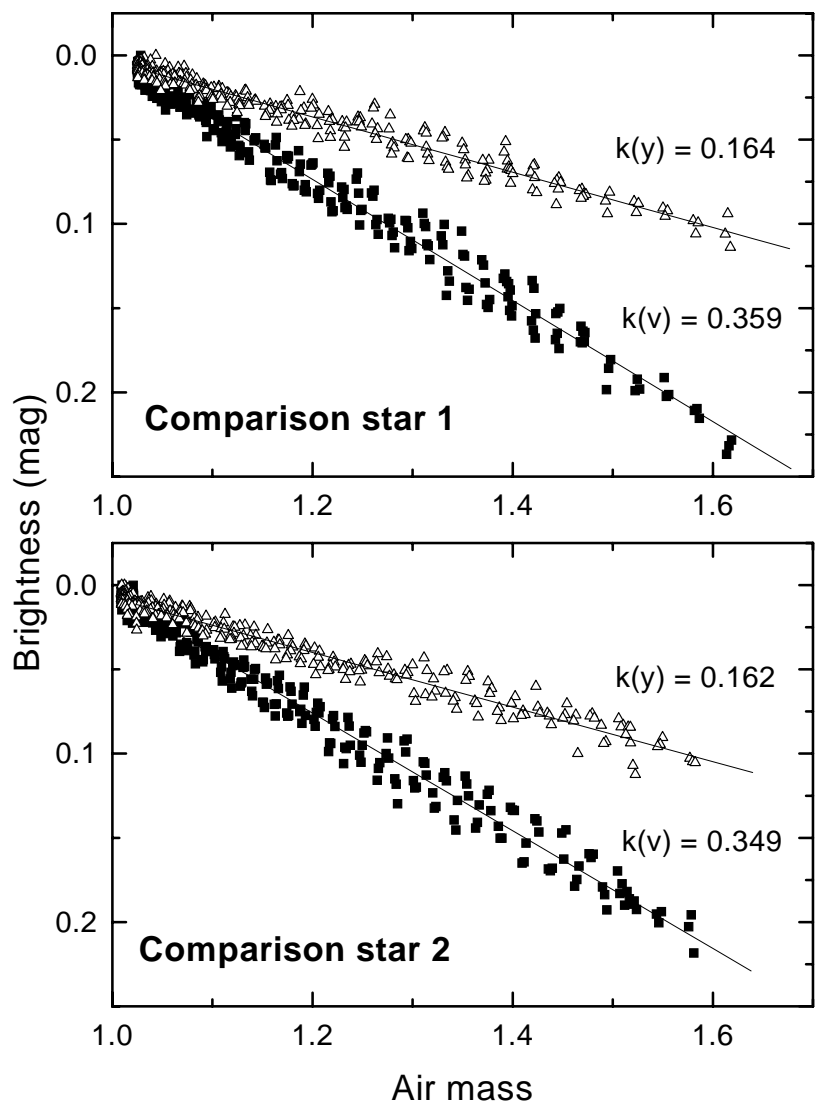

Fig. 1. Extinction diagrams for the night of 1997 March 19. The diagram demonstrates the high accuracy with which the extinction coefficients could be determined due to the large number of measurements

The conventional technique provides a heavy burden on the observer: the large amount of required data means work of weeks or months at an observatory, which can be quite expensive. Our method of observation with the three-star technique is perfectly suited for an Automatic Photoelectric Telescope (APT), if sufficient accuracy can be obtained without observer intervention. The present 
paper reports a successful application of APT technology to $\delta$ Scuti stars.

The variability of the $\delta$ Scuti star 4 CVn $(\mathrm{HR} 4715=$ HD $107904=$ AI CVn, F3III-IV) was discovered by Jones \& Haslam (1966). The discovery was followed by a number of usually relatively short observational studies with contradictory frequency solutions caused, in part, by the lack of data. During the years 1983 and 1984 a multisite campaign by the Delta Scuti Network (Breger et al. 1990, Paper I) was carried out at four collaborating observatories. This led to the determination of five pulsation frequencies which are free of $1 \mathrm{~cd}^{-1}$ alias problems. The analysis of the campaign data together with the previously (mostly unpublished) photometry revealed that $4 \mathrm{CVn}$ pulsates with at least seven frequencies with values of $8.59,7.37,6.98,6.19,5.85,5.53$, and $5.05 \mathrm{~cd}^{-1}$. These seven frequencies are independently found and confirmed in different subsets of the 114 nights of photometric data covering the years 1966 to 1984 (Breger 1990a, Paper II).

Extensive series of unpublished photometry (see Fitch 1980) also showed two unusually small frequencies. 288 hours of new high-quality observations during a 58day time span were obtained by Breger et al. (1997) to solve this question. The two low frequencies of 1.32 and $1.40 \mathrm{~cd}^{-1}$ were found to originate in the comparison star used in many studies, HD 108100, which was hereby discovered to be a $\gamma$ Doradus g-mode pulsator.

\section{Photometry with the APT}

The observations were carried out with one of the two $0.75 \mathrm{~m}$ APTs located at Washington Camp in Arizona, USA. Since 1996, the twin telescopes named "Wolfgang" and "Amadeus" are the property of the University of Vienna. A detailed description of these telescopes can be found in the paper by Strassmeier et al. (1997). In this paper it was reported that for variable stars, a photometric accuracy of $\pm 4 \mathrm{mmag}$ in $b$ and $y$ had been achieved. This suggests that with some additional improvement of the accuracy, the telescopes could be used for the study of $\delta$ Scuti stars.

The configuration used for the present observations with the Wolfgang telescope is a Cassegrain system with a focal ratio of $\mathrm{f} / 8$. The detector system consists of a $800 \times 490$ pixel CCD used for centering the object. The actual data aquisition is performed with a blue-sensitive bi-alkali EMI-9124/QB PMT. The water-cooled tube is operated at a temperature of $3^{\circ} \mathrm{C}$ and has a typical dark count of $20 \mathrm{cs}^{-1}$.

The outstanding advantage of using an APT for our three-star method is the telescope's speed. The acquisition of the navigation star, which is needed for finding the actual target group, is performed with a slew speed of $10^{\circ} \mathrm{s}^{-1}$ at a general pointing accuracy of 30 arcsec. Once this star has been found, the time needed to slew between

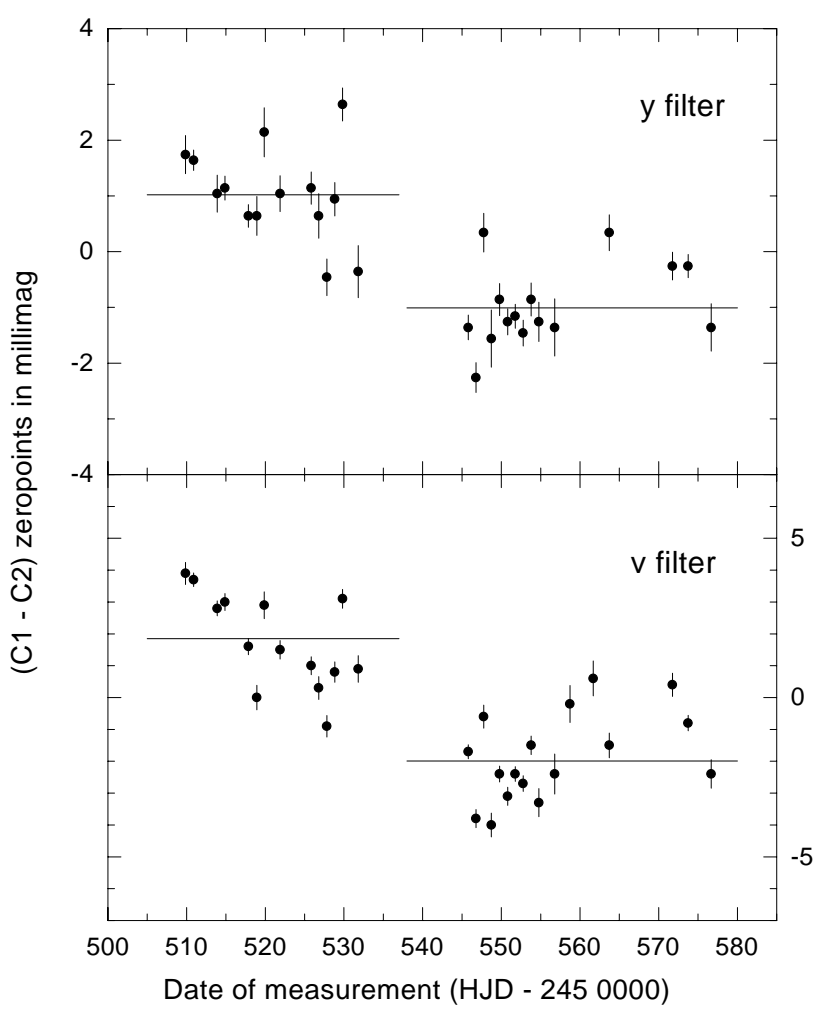

Fig. 2. Variation of brightness difference between the two comparison stars (normalized to zero). The error bars are based on the scatter of the individual measurements within a night. The slow variation is probably a consequence of instrumental variations of the APT and not due to small variability of one of the comparison stars (see text)

the stars of the group and to center the star is about $1 \mathrm{~s}$, which is fast compared to the time the human observer needs for finding and centering the next object. For this reason the APT can be regarded as ideal instrument for time-series photometry, making it possible to obtain more than 300 separate measurements during a good night.

On the other hand, some problems specific to the APT were found to exist:

- Difficulties in finding and centering the objects, especially if the weather conditions are not ideal. The APT recognizes objects by their brightness. An average brightness value is given and the telescope measures and compares the objects in question with the preset value. If cloudy or hazy weather influences the brightness of a star, misidentifications may occur. Such problems are, of course, recognized from the data.

- No other stellar object within 0.5 mag (in Johnson $B$ ) of the target's brightness may be in the finder field (about 9 arcmin), as this may make it difficult for the centering algorithm to choose the correct target.

- Another problem concerns the lack of the clear error messages coming from the telescopes. This makes it hard for the observer to pinpoint the source of possible problems. For example, the message "Object not found" could be the result of bad weather, wrong 
Table 1. Journal of the APT measurements of $4 \mathrm{CVn}$

\begin{tabular}{|c|c|c|c|c|c|c|}
\hline \multicolumn{2}{|c|}{ Date (Beginning) } & \multirow{2}{*}{$\begin{array}{l}\text { Length } \\
\text { hours }\end{array}$} & \multicolumn{2}{|c|}{$\mathrm{C} 1-\mathrm{C} 2$ residuals } & \multicolumn{2}{|c|}{ Extinction coefficients } \\
\hline UT & HJD (245 $0000+)$ & & $y$ (mmag) & $v$ (mmag) & $k_{y}$ & $k_{v}$ \\
\hline 970302 & 509.720 & 6.2 & 2.4 & 2.5 & 0.14 & 0.33 \\
\hline 970303 & 510.714 & 8.0 & 1.9 & 2.2 & 0.12 & 0.30 \\
\hline 970306 & 513.755 & 6.8 & 2.9 & 2.0 & 0.16 & 0.35 \\
\hline 970307 & 514.705 & 7.6 & 2.1 & 2.7 & 0.16 & 0.36 \\
\hline 970310 & 517.694 & 7.1 & 2.0 & 2.5 & 0.18 & 0.37 \\
\hline 970311 & 518.845 & 4.4 & 2.7 & 2.9 & 0.17 & 0.36 \\
\hline 970312 & 519.757 & 5.2 & 2.6 & 2.6 & 0.12 & 0.33 \\
\hline 970313 & 520.722 & 7.5 & (1) & & 0.16 & 0.38 \\
\hline 970314 & 521.714 & 7.4 & 2.7 & 2.5 & 0.16 & 0.34 \\
\hline 970318 & 525.674 & 8.3 & 2.8 & 2.7 & 0.16 & 0.35 \\
\hline 970319 & 526.670 & 6.2 & 3.2 & 2.9 & 0.16 & 0.35 \\
\hline 970320 & 527.668 & 8.1 & 3.1 & 3.0 & 0.17 & 0.36 \\
\hline 970321 & 528.680 & 7.9 & 2.8 & 3.0 & 0.17 & 0.37 \\
\hline 970322 & 529.662 & 7.6 & 2.6 & 2.6 & 0.14 & 0.33 \\
\hline 970324 & 531.664 & 7.3 & 3.2 & 2.8 & 0.15 & 0.33 \\
\hline 970407 & 545.618 & 8.2 & 2.3 & 2.3 & 0.16 & 0.33 \\
\hline 970408 & 546.615 & 8.3 & 2.8 & 3.0 & 0.17 & 0.38 \\
\hline 970409 & 547.624 & 6.3 & 3.2 & 3.1 & 0.15 & 0.24 \\
\hline 970410 & 548.625 & 5.2 & 2.9 & 2.9 & 0.20 & 0.39 \\
\hline 970411 & 549.616 & 8.1 & 3.0 & 2.6 & 0.19 & 0.38 \\
\hline 970412 & 550.657 & 7.0 & 2.1 & 2.6 & 0.18 & 0.37 \\
\hline 970413 & 551.617 & 8.0 & 2.2 & 2.4 & 0.19 & 0.40 \\
\hline 970414 & 552.618 & 7.8 & 2.1 & 2.3 & 0.17 & 0.36 \\
\hline 970415 & 553.622 & 7.6 & 2.6 & 2.6 & 0.17 & 0.36 \\
\hline 970416 & 554.673 & 4.6 & 2.5 & 3.1 & 0.22 & 0.42 \\
\hline 970418 & 556.688 & 4.2 & 2.9 & 3.5 & 0.17 & 0.41 \\
\hline 970420 & 558.639 & 4.7 & (1) & 3.9 & 0.16 & 0.38 \\
\hline 970423 & 561.622 & 2.5 & (1) & 2.8 & & 0.28 \\
\hline 970425 & 563.623 & 4.6 & 2.3 & 2.8 & 0.25 & 0.44 \\
\hline 970503 & 571.670 & 4.0 & 1.8 & 2.7 & 0.15 & 0.31 \\
\hline 970505 & 573.632 & 6.1 & 1.9 & 2.2 & 0.14 & 0.31 \\
\hline 970508 & 576.633 & 1.6 & 2.0 & 2.1 & & \\
\hline
\end{tabular}

(1) Only comparison star C1 used.

input data to the telescope, technical problems of the telescope or its steering, or a number of other possible reasons.

- The present operating procedure of the APT limits the maximum hour angle to 4.2 hours. This means that the longest set of observations of $4 \mathrm{CVn}$ could only cover slightly more than 8 hours.

These difficulties can be attributed to the operation program ATIS (Automatic Telescope Instruction Set) controlling the telescope. A new, improved version has been developed by NASA engineers and is currently undergoing the first tests at the APT.

The current limitations of the telescope render it unusable for certain applications such as observations in crowded fields, since the centering algorithm of the instrument might become confused. This does not present a problem for the present program reported in this paper. We also note that APT measurements should not be obtained within $30^{\circ}$ of the Moon.
Between 1997 March 2 and May 8, more than 40 nights of photometric data of $4 \mathrm{CVn}$ were collected with the Wolfgang APT telescope. $4 \mathrm{CVn}$ and two comparison stars were measured through the Stromgren $y$ and $v$ filters. The data were reduced in the standard photometric manner. The comparison stars used were HR 4728 (G9III) and HR 4843 (F6IV), for which no variability had been detected during previous campaigns. Each observation consisted of three single 10-s integrations.

A difference between measurements obtained automatically and manually by an observer lies in the handling of measurements obtained during marginal weather conditions. While an observer might stop or attach notes to marginal measurements, the quality of APT measurements need to be judged afterwards. This quality control is not available for the present program: instead we rejected all measurements for which the standard deviation of the three single $10 \mathrm{~s}$ integrations was $\geq 10 \mathrm{mmag}$. Furthermore, all nights or longer fraction of nights during 

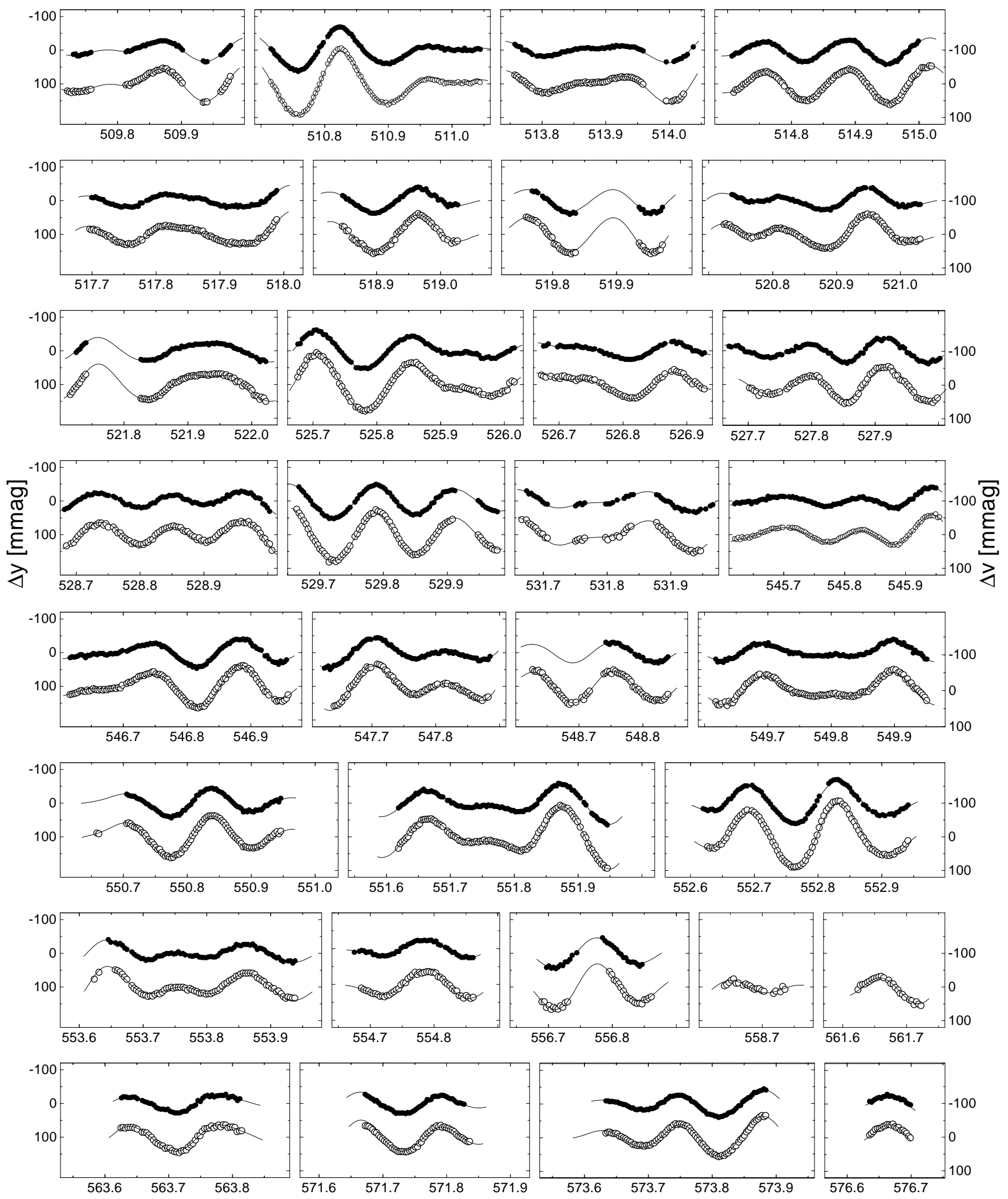

Fig. 3. APT photometry of 4 CVn obtained during the 1997 APT campaign. $\Delta y$ and $\Delta v$ are defined to be the magnitude differences (variable-comparison stars) normalized to zero. The fit of the 19-frequency solutions derived in this paper is shown as a solid curve 

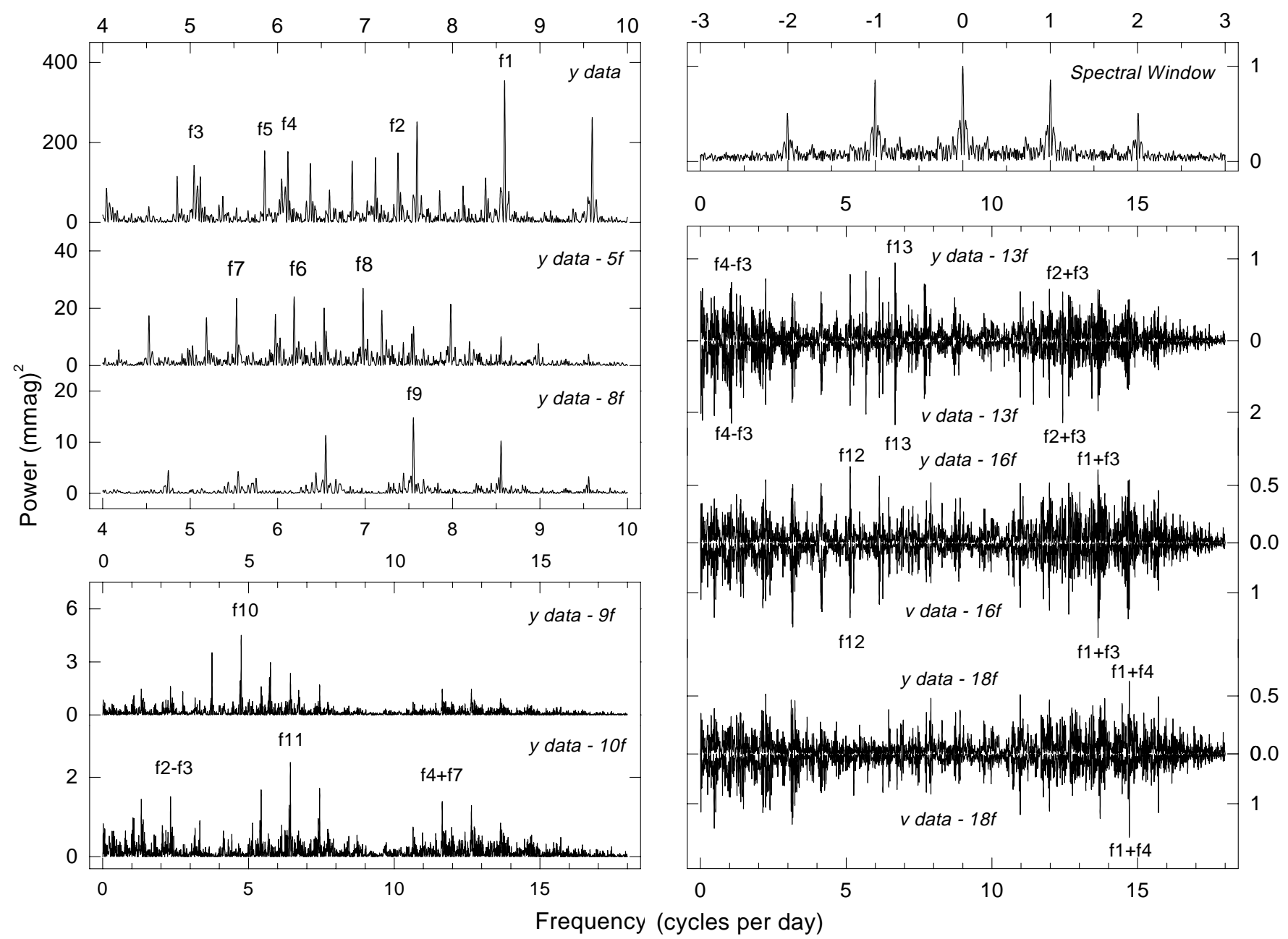

Fig. 4. Power spectrum of the 1997 photometry of $4 \mathrm{CVn}$. The different panels present the results after prewhitening a given number of previously detected frequencies. For the 13 main modes the $y$ and $v$ results are similar so that only $y$ data are shown. For the additional modes, $y$ and $v$ (plotted inverted) data are presented separately at the lower right

which the standard deviation of the $\mathrm{C} 1-\mathrm{C} 2$ measurements was larger than $4.0 \mathrm{mmag}$ were rejected. We were left with high-quality data covering 204 hours during 32 nights (see Table 1). Most of the observing time, which could not be used or kept after reduction, was due to unfavorable weather conditions rather than equipment problems.

Table 1 also lists the extinction coefficients which were derived from the two comparison stars for each night. The agreement between the coefficients derived from the two comparison stars is demonstrated in Fig. 1 for the night of 1997 March 19. From the residuals of $\mathrm{C} 1-\mathrm{C} 2$ listed in Table 1, it can be seen that the night is of average quality. The data also show a small, systematic variation in extinction between the Eastern and Western hemispheres. This effect can also be seen on a few other nights, but with different signs. Consequently, a systematic East/West extinction difference cannot be demonstrated at this stage. These differences are too small to produce any detectable effect on the differential photometry of $4 \mathrm{CVn}$. We have also checked the extinction coefficients derived in this standard way by computing differential extinction coefficients between $\mathrm{C} 1-\mathrm{C} 2$. The agreement with the values determined in the standard way was excellent.

It might be interesting to look at the average extinction coefficients during the campaign. If we restrict ourselves to nights with $\geq 3.0$ hours of observation and exclude the night of 970425 , which clearly has higher extinction values, the extinction coefficients for the remaining 29 nights show a Gaussian distribution. The following average values are obtained:

$k_{y}=0.163 \pm 0.021$, and $k_{v}=0.353 \pm 0.037$

These values are typical for the site. Further measurements are needed to judge whether or not the observed variations of the coefficients are significant.

Figure 2 shows that the magnitude difference between $\mathrm{C} 1$ and $\mathrm{C} 2$ exhibits a small, systematic drift over the nine weeks. The drift in $v$ is about twice the amount in $y$. The most natural explanation would be small variability of one of the two comparison stars. Since the third star observed, $4 \mathrm{CVn}$, is not expected to show such slow variability, it 
Table 2. Pulsation frequencies and amplitudes of $4 \mathrm{CVn}$

\begin{tabular}{ccccccc}
\hline \multicolumn{2}{c}{ Frequency } & \multicolumn{2}{c}{1997 amplitudes } & \multicolumn{2}{c}{ Signal/Noise } & $1966-1984$ amplitudes \\
& cd $^{-1}$ & $y$ & $v$ & $y$ & $v^{(1)}$ & $y$ \\
& & mmag & mmag & & & mmag \\
\hline$f_{1}$ & 8.595 & 17.3 & 25.9 & 81 & 85 & $0-14$ \\
$f_{2}$ & 7.375 & 12.0 & 18.1 & 61 & 62 & $4-25$ \\
$f_{3}$ & 5.048 & 11.6 & 17.7 & 58 & 54 & Newly grown mode \\
$f_{4}$ & 6.117 & 10.4 & 15.8 & 53 & 52 & $8-12$ \\
$f_{5}$ & 5.851 & 10.3 & 15.3 & 52 & 50 & $0-6$ \\
$f_{6}$ & 6.191 & 5.4 & 8.3 & 28 & 27 & $0-12$ \\
$f_{7}$ & 5.532 & 5.1 & 8.3 & 25 & 26 & New detection \\
$f_{8}$ & 6.976 & 4.9 & 7.3 & 25 & 25 & New detection \\
$f_{9}$ & 7.552 & 4.4 & 6.1 & 22 & 21 & New detection \\
$f_{10}$ & 4.749 & 2.2 & 3.7 & 11 & 11 & New detection \\
$f_{11}$ & 6.440 & 1.9 & 3.3 & 10 & 11 & $=f_{4}+f_{7}$, new detection \\
$f_{12}$ & 5.135 & 1.1 & 1.6 & 5.3 & 4.9 & $f_{2}-f_{3}$, new detection \\
$f_{13}$ & 6.680 & 1.0 & 1.5 & 4.9 & 4.9 & $=f_{4}-f_{3}$, new detection \\
$f_{14}$ & 11.649 & 1.1 & 1.7 & 4.6 & 5.0 & $=f_{1}+f_{3}$, new detection \\
$f_{15}$ & 2.327 & 1.1 & 1.6 & 4.2 & 3.6 & $=f_{1}+f_{4}$, new detection \\
$f_{16}$ & 1.069 & 0.9 & 1.7 & 3.7 & 4.1 & $=f_{2}+f_{3}$, new detection \\
$f_{17}$ & 13.643 & 0.8 & 1.5 & 3.3 & 4.3 & \\
$f_{18}$ & 14.712 & 0.8 & 1.3 & 3.3 & 4.0 & \\
$f_{19}$ & 12.423 & 0.7 & 1.3 & 2.6 & 3.6 & \\
\hline
\end{tabular}

(1) Amplitude signal/noise limit is 4.00 for newly discovered pulsation modes and 3.50 for "expected" combination frequencies.

should be possible to determine which of the two comparison stars might be variable. An examination of the zero-point residuals of $4 \mathrm{CVn}$ (after the fits in the next section) shows similar drifts in both $\mathrm{V}-\mathrm{C} 1$ and $\mathrm{V}-\mathrm{C} 2$, but with different amplitudes. This suggests an instrumental origin of the drifts.

We note the possible existence of low-frequency stability problems of the APT in the millimag range, which do not seriously affect the reductions of the much more rapidly varying star $4 \mathrm{CVn}$. For the reduction of $4 \mathrm{CVn}$, we have represented the drift between the two comparison stars by two different values of $(\mathrm{C} 1-\mathrm{C} 2)$ in each filter (shown as straight lines in the figure.) The twoweek gap in the middle of the observations were caused by poor weather conditions, rather than equipment problems. Consequently, the agreement between the observing gap and the drift maximum is probably accidental. The power spectrum of $\mathrm{C} 1-\mathrm{C} 2$ shows a peak of $0.7 \mathrm{mmag}$ at $0.036 \mathrm{~cd}^{-1}$ in $v$ and $0.7 \mathrm{mmag}$ at $0.79 \mathrm{~cd}^{-1}$ in $y$. Consequently, any detected low frequencies in program stars observed with the APT should be checked whether they are shown in all filters and relative to all comparison stars.

The following residuals were found for $\mathrm{C} 1-\mathrm{C} 2$ : $\pm 2.7 \mathrm{mmag}$ for $y$ and $\pm 3.0 \mathrm{mmag}$ for $v$ per single measurement.

The light curves of $4 \mathrm{CVn}$ are shown in Fig. 3.

\section{Multiperiodicity and amplitude variability of $4 \mathrm{CVn}$}

The pulsation frequency analyses were performed with a package of computer programs with single-frequency and multiple-frequency techniques (programs PERIOD, Breger 1990b; PERIOD98, Sperl 1998), which utilize Fourier as well as multiple-least-squares algorithms. The latter technique fits a number of simultaneous sinusoidal variations in the magnitude domain and does not rely on prewhitening. For the purposes of presentation, however, prewhitening is required if the low-amplitude modes are to be seen. Therefore, the various power spectra are presented as a series of panels in Fig. 4, each with additional frequencies removed relative to the panel above. The $v$ and $y$ data give identical results for the first 13 frequencies so that only the $y$ results are presented in the left panel. For the additional frequencies, we present both the $y$ and $v$ (inverted) results in the right panel. Note that for the detected frequencies, the amplitude ratio, $v / y$, is near 1.5, as expected for these stars. This increases the confidence that the detected frequency peaks correspond to real pulsation modes, rather than noise artifacts.

One of the most important questions in the examination of multiperiodicity concerns the decision as to which of the detected peaks in the power spectrum can be regarded as variability intrinsic to the star. Due to the presence of nonrandom errors in photometric observations and because of observing gaps the predictions of standard statistical false-alarm tests give answers which are considered by us to be overly optimistic. In a previous paper (Breger et al. 1993) we have argued that a ratio of amplitude 
signal $/$ noise $=4.0$ provides a useful criterion for judging the reality of a peak for multisite data. This criterion can be somewhat relaxed for peaks at previously expected values; viz. harmonics or linear combination frequencies, where a value of 3.5 is adopted. In the present study the noise was calculated by averaging the amplitudes (oversampled by a factor of 20) over $5 \mathrm{~cd}^{-1}$ regions centered around the frequency under consideration. For the two combination frequencies below $3 \mathrm{~cd}^{-1}$, smaller intervals of $1 \mathrm{~cd}^{-1}$ were chosen. The results are shown in Table 2, which also compares the new results to those found by reanalyzing the available data in the literature for the years from 1966 to 1984.

We noted earlier that the low-frequency terms found in APT data need to be considered with some caution. For $4 \mathrm{CVn}$, two low frequencies were detected. We regard their reality as quite probable since these peaks do not show up in the $\mathrm{C} 1-\mathrm{C} 2$ data and the values of the detected frequencies match the values calculated from frequency combinations exactly.

The average deviation of the observations from the fit are $3.0 \mathrm{mmag}$ per single measurement in $y$ and $4.0 \mathrm{mmag}$ in $v$. This makes it possible to estimate the uncertainties of the amplitudes shown in Table 2. Based on the assumption that these residuals are random, we can apply the equation $\sigma(a)=\sigma(m)(N / 2)^{-1 / 2}$, where $a$ is the amplitude, $\sigma(m)$ is the average residual of each data point, and $N$ the number of measurements. We derive uncertainties of \pm 0.09 and \pm 0.12 mmag for the $y$ and $v$ amplitudes, respectively. Of course, in reality the sources of error are neither random nor independent of frequency (white noise). It is interesting to note that combining the two $y, v$ data sets does not lower the noise level significantly and cannot improve the mode detection. Inspection of the data suggests two reasons: the computed noise in the frequency region under discussion is composed mainly of undetected additional modes and the measuring errors of $y$ and $v$ are not independent of each other. Although it is not possible to evaluate the errors in more detail, the present calculation can be useful to estimate whether or not observed amplitude variability is real.

\section{Additional discussion}

The APT measurements have increased the number of known pulsation frequencies of $4 \mathrm{CVn}$ tremendously from 7 to 19 , of which 6 are linear combination frequencies. All of the previously detected frequencies were confirmed with high amplitudes of $5 \mathrm{mmag}$ or more. The newly detected frequencies have smaller amplitudes, except for $f_{4}$, which has appeared with a very high amplitude of $10 \mathrm{mmag}$ in $y$ and $16 \mathrm{mmag}$ in $v$. Although $f_{6}$ and $f_{4}$ are separated by only $0.07 \mathrm{~cd}^{-1}$, the resolution of the older data in the literature is sufficient to separate the two modes unambiguously. Inspection of the older data indicates that this pulsation mode was either not present earlier or had an amplitude of 3 mmag or less.

This growth of a "new" pulsation mode is not unusual for $4 \mathrm{CVn}$. The star has a history of slow but steady amplitude changes with a time scale of years (see Breger 1990a). Nevertheless, more spectacular changes also occur: from 1974 to $1976 / 7$, the high-amplitude mode $f_{2}$ essentially disappeared in order to slowly grow again in amplitude during the next decade.

It was shown that the presently available APT is able to measure at the millimag level. For differences, C1-C2, in the $y$ and $v$ filters, residuals of 2.65 and $3.00 \mathrm{mmag}$ were found, respectively. 32 nights of data of the $\delta$ Scuti variable $4 \mathrm{CVn}$ made it possible to extract 19 simultaneously excited frequencies with amplitudes as small as $1 \mathrm{mmag}$. The 19-frequency fit of $4 \mathrm{CVn}$ leaves residuals of 2.98 and 4.03 mmag. These residuals are slightly higher than those of the comparison stars and indicate the presence of additional pulsation modes with small amplitudes. The presence of additional, undetected modes is not in contradiction to the excellent fit of the observed and computed light curves shown in Fig. 3. The visible light curve is comprised mainly of the modes with large amplitudes, viz. $f_{1}$ to $f_{8}$. Large amounts of high-precision data allow the extraction of additional modes whose detection is statistically significant while not changing the quality of the computed light curves to a noticeable degree.

Acknowledgements. Part of the investigation has been supported by the Austrian Fonds zur Förderung der wissenschaftlichen Forschung, project numbers S7304 and S7301.

\section{References}

Breger M., 1990a, A\&A 240, 308 (Paper II)

Breger M., 1990b, Comm. Asteroseismology (Vienna) 20, 1

Breger M., 1993, in Stellar Photometry - Current Techniques and Future Developments (IAU Coll. 136), Butler C.S. \& Elliott I. (eds.). Cambridge Univ. Press, p. 106

Breger M., Handler G., Garrido R., et al., 1997, A\&A 324, 566

Breger M., McNamara B.J., Kerschbaum F., et al., 1990, A\&A 231, 56 (Paper I)

Breger M., Pamyatnykh A.A., Pikall H., Garrido R., 1998, A\&A (in press)

Breger M., Stich J., Garrido R., et al., 1993, A\&A 271, 482

Fitch W.S., 1980, Lect. Notes Phys. 125, 7

Guzik J.A., Templeton M.R., Bradley P.A., 1998, ASPC 135, 470

Jones D.H.P., Haslam C.M., 1966, The Observatory 86, 34

Pamyatnykh A.A., Dziembowski W.A., Handler G., Pikall H., 1998, A\&A 333, 141

Sperl M., 1998, Comm. Asteroseismology (Vienna) 111, 1

Strassmeier K.G., Boyd L.J., Epand D.H., Granzer Th., 1997, PASP 109, 697

Viskum M., Kjeldsen H., Bedding T.R., et al., 1998, A\&A 335, 549 